\title{
CLINICAL CORRELATION BETWEEN ANTI-CCP ANTIBODY, RHEUMATOID FACTOR AND CLINICAL PARAMETERS OF PATIENTS WITH RHEUMATOID ARTHRITIS
}

\author{
Vaidya B. ${ }^{1}$, Nakarmi S. ${ }^{2}$, Rajbhandari N. ${ }^{3}$, Bataju P.
}

\begin{abstract}
BACKGROUND: The main clinically useful serological markers in patients with Rheumatoid Arthritis are Rheumatoid Factor and Anti-citrullinated Protein Antibodies (ACPA) for both diagnosis and prediction of functional and radiographic outcomes. We do not have data from Nepalese patients about gender differences and association of these antibodies with disease nature.
\end{abstract}

OBJECTIVE: To identify the difference in nature of disease in relation to ACPA and gender.

METHODS: A cross sectional open labeled study including consecutive patients who were diagnosed as RA according to ACR/EULAR 2010 attending the rheumatology OPD from October 2010 to October 2012 at Alka Hospital, Nepal. Data collection was done by direct patient interview and all data were recorded electronically in a predesigned performa. ACPA and RF were tested in the serum samples of 184 patients.

RESULTS: Out of 184 patients, 114 of them were ACPA positive and 54 were Rheumatoid factor positive. The median duration of symptoms was $30.3 \pm 7.0$ months lesser in ACPA positive patients as compared to negative ones. Mean age of females was 10.7 years less than male patients. No other significant differences were noted between male and female patients. Also, $52.3 \%$ of RF negative patients were found to have ACPA positivity.

CONCLUSION: Anti cyclic citrullinated peptide antibodies are excellent serological markers of rheumatoid arthritis, which shows good diagnostic value. This can increase the diagnostic value in addition to rheumatoid factor and its use in clinical practice may contribute to increased ability of rheumatologists to make judicious treatment decisions.

KEYWORDS: Anti-Cyclic Citrullinated Peptide Antibodies, Rheumatoid Arthritis, Rheumatoid Factor.

1. Physician, Alka Hospital, Kathmandu, Nepal

2. Research officer, Alka Hospital, Kathmandu, Nepal

3. Medical Officer, Nepal Medical College, Kathmandu, Nepal

For Correspondence

Dr. BinitVaidya, M.D., F.A.C.R. Physician,

Department of Medicine

Alka Hospital, Kathmandu, Nepal

Email: drbinitvaidya@yahoo.com 


\section{INTRODUCTION}

Rheumatoid Arthritis (RA) is a multisystem autoimmune disease characterized by progressive involvement of small and large joints leading to deformities. Diagnosis of RA is made primarily on clinical manifestations and serum biomarkers, of which serum Rheumatoid Factor (RF) is frequently used. However, the sensitivity of RF is approximately 60 to $80 \%$ in RA, and the specificity is also low since RF is detectable in many other diseases and in approximately $5 \%$ of healthy people. ${ }^{1,2}$ More recently a highly specific ACPA has been identified. The second generation of these antibody tests (anti CCP2) has been found to have a sensitivity of $68 \%$ and a specificity $>97 \% .^{3-5}$ Anti-CCP antibodies have also demonstrated prognostic utility with regard to radiographic outcomes.

The present study was designed to examine the sensitivity of anti-CCP antibody in diagnosis of RA and the relationship between anti-CCP antibodies and RF in a diagnosed RA patient.

Also many researchers have shown gender differences in RA. ${ }^{7,8}$ Other than the higher prevalence in female patients, studies have shown that male patients with RA tend to have aggressive disease. ${ }^{9}$ This study also aimed to identify the difference in disease nature with respect to gender as well as relation to ACPA.

\section{METHODS}

This cross sectional open labeled observational study included consecutive patients who were diagnosed as Rheumatoid Arthritis attending the rheumatology OPD from October 2010 to October 2012. Diagnosis of RA was made according to 2010 EULAR/ACR criteria ${ }^{10}$ and a sample size of 184 with power of study of $95 \%$ and a significance level of 0.05 was taken.

Clinical details of all the patients along with the serology reports were entered electronically in a predesigned excel sheet. Clinical details included duration of disease process, pattern of joint involvement, early morning stiffness, extra articular and systemic involvement in addition to the demographic data. RF and second generation anti CCP antibodies were tested using ELISA method. Other relevant reports of CRP, ESR etc were also recorded.

Patients with other autoimmune inflammatory diseases like SLE, Sjogrens, reactive arthritis, psoriatic arthritis etc. as primary diagnosis were excluded from the study. However, those with overlap syndromes with RA as a component were still included.

Student t-test and independent sample t tests were used to determine differences in groups. Chi square test was used to determine significance of differences in categorical variables. Fisher exact test was used to measure associations of ACPA with various variables. Statistical analyses were done using SPSS 19

\section{RESULTS}

A total of 184 patients were analyzed. Of all patients, $163(88.59 \%)$ were females with male:female ratio of approximately 1:7. The mean age of our patients was $46.5 \pm$ 13.4 (11-86) years and median duration of disease was 24 months.

The mean age and disease duration of male and female patients are shown in table 1 .

Table 1: Difference between male and female patients with RA

\begin{tabular}{|l|l|l|l|l|l|}
\hline & Gender & $\mathrm{N}$ & Mean & Std. Deviation & P value \\
\hline \multirow{2}{*}{ Age } & Female & 163 & 45.3 & 13.0 & 0.001 \\
\cline { 2 - 6 } & Male & 21 & 56.0 & 13.6 & \\
\hline \multirow{2}{*}{ Duration } & Female & 163 & 44.6 & 48.9 & 0.90 \\
\cline { 2 - 6 } & Male & 21 & 45.9 & 49.7 & \\
\hline
\end{tabular}

It was seen that the mean age of female patients was less than that of male patients by an average of approximately $10.74 \pm 5$ years $[p=0.001]$. There was no significant difference in other clinical and laboratory parameter between male and female patients including the positivity for ACPA and RF (table 2). Relationship of clinical parameters with ACPA:

Table 2: Difference between male and female patients (other parameters)

\begin{tabular}{|l|l|l|l|}
\hline Parameter & Male (/21) & Female (/163) & P value \\
\hline Small joint & 19 & 149 & 0.53 \\
\hline Large joint & 16 & 139 & 0.67 \\
\hline EMS & 12 & 92 & 0.81 \\
\hline Sacroilietis & 3 & 5 & 0.34 \\
\hline Deformities & 13 & 25 & 0.40 \\
\hline RF positive & 5 & 49 & 0.62 \\
\hline ACPA positive & 14 & 100 & 0.81 \\
\hline Duration (months) & 45.9 & 44.6 & 0.91 \\
\hline
\end{tabular}

Out of 184 patients, 114 (61.9\%) of them were Anti-CCP positive(100 females, 14 males: $p=0.63$ ). The mean age and disease duration of RA patients in relation to ACPA positivity are shown in table 3 . 
Table 3: Relationship of ACPA with age and duration of disease.

\begin{tabular}{|l|l|l|l|l|l|l|}
\hline \multirow{2}{*}{ Duration } & ACPA & N & Mean & $\begin{array}{c}\text { Std. } \\
\text { Deviation }\end{array}$ & $\begin{array}{c}\text { Std. Error } \\
\text { Mean }\end{array}$ & P value \\
\cline { 2 - 7 } & Negative & 70 & 63.60 & 47.110 & 5.631 & $<0.001$ \\
\cline { 2 - 7 } & Positive & 114 & 33.24 & 46.484 & 4.354 & \\
\hline \multirow{2}{*}{ Age } & Negative & 70 & 46.09 & 12.812 & 1.531 & 0.72 \\
\cline { 2 - 7 } & Positive & 114 & 46.82 & 13.945 & 1.306 & \\
\hline
\end{tabular}

The median duration of disease was $30.3 \pm 7.0$ months lesser in ACPA positive cases $[p=<0.001]$. Similar relationship with disease duration was not seen with RF positivity $[p=0.65]$. ACPA positivity did not associate with any other clinical or laboratory parameters tested (table 4).

Table 4: Relationship with ACPA (other parameters)

\begin{tabular}{|l|l|l|l|}
\hline Parameter & Positive & Negative & P value \\
\hline Small joint & 100 & 64 & 0.25 \\
\hline Large joint & 76 & 50 & 0.43 \\
\hline EMS & 108 & 62 & 0.23 \\
\hline Sacroilietis & 4 & 2 & 0.34 \\
\hline Deformities & 12 & 5 & 0.90 \\
\hline
\end{tabular}

As expected, RF and ACPA positivity showed significant positive association $\left[\chi^{2}=17.5 ; \mathrm{p}=<0.001\right]$. Out of $130 \mathrm{RF}$ negative patients $68(52.3 \%)$ were ACPA positive. Both AntiCCP and RF were negative in 62(33.6\%) patients.

Table 5: Relationship between RF and ACPA(Chi Square)

\begin{tabular}{|c|l|l|l|l|}
\hline \multicolumn{2}{|c|}{} & \multicolumn{2}{c|}{ RF } & \multirow{2}{*}{ Total } \\
\cline { 3 - 5 } \multicolumn{2}{|c|}{ Neg } & \multicolumn{1}{c|}{ Pos } & \\
\hline \multirow{2}{*}{ ACPA } & Negative & 62 & 8 & 70 \\
\cline { 2 - 5 } & Positive & 68 & 46 & 114 \\
\hline \multicolumn{2}{|c|}{ Total } & 130 & 54 & 184 \\
\hline
\end{tabular}

\section{DISCUSSION}

RA is a systemic autoimmune disease with more prevalence in females. Studies have shown mixed results on the nature of disease in male patients. Many prior studies have shown later age at onset of RA in male patients with $\mathrm{RA}^{7-9}$ and same findings are replicated in our study too. It seems that disease onset in females was either earlier or the disease might be severe for earlier presentation of these patients. However, once the disease is present, the clinical features like pattern of joint involvement, systemic involvement and serological features were similar in male and female patients in our study. In contrast, a study in 2001 has shown greater occurrence of RF positivity in males. ${ }^{8}$ Moreover, male patients were seen to have more erosive disease and more positivity for RF and ACPA and had higher titres of these antibodies . ${ }^{9}$ The authors have associated these findings to shared epitope in familial cases. Our study does not confirm to these findings which might be due to different genetic constitution of Nepalese patients and also may be due to the use of ACR/EULAR 2010 criteria $^{10}$ for diagnosis. Earlier diagnosis of male patients might have influenced the course and outcome in these patients. Gender differences in disease severity and outcome must be reassessed using the new criteria for diagnosis.

RA is a chronic disease process and it is now proven beyond doubt that the earlier the diagnosis and treatment, the better the prognosis. For many years, RF was the only serological marker available for the supportive diagnosis of RA. However, with the growing popularity of ACPA, RA is being diagnosed earlier. ${ }^{11,12}$ Studies have shown that presence of ACPA indicates more aggressive disease. ${ }^{13,14}$ Studies have correlated ACPA with DAS, CRP and RF. ${ }^{15}$ In our study, we found that presence of ACPA was associated with short disease duration than those with absence of ACPA. This could also mean aggressive disease leading to early presentation in these patients. However, there was no age or gender difference in relation to ACPA. We did not find any significant difference in presence of erosions or deformities in patients with ACPA positivity in contrast to previous studies. ${ }^{13,14}$ This is due to cross sectional nature of study and early diagnosis due to use of new criteria. A long term follow up of these patients might show findings consistent with prior studies.

The sensitivity of ACPA of $61.9 \%$ nearly matches that in prior studies of 56 to $64 \% .{ }^{15-17}$ An important finding however was $52.3 \%$ diagnosed as seropositive with the use of ACPA who would otherwise be deemed seronegative with the use of RF only. Addition of ACPA increased the sensitivity of diagnosis of RA in RF negative patients.

\section{CONCLUSION}

Female patients with RA had significantly lower age than male patients. The disease did not differ much in terms of presence of RF, ACPA, erosions or other clinical parameters between male and female patients. Also, ACPA positivity was seen in approximately $62 \%$ of our patients. Use of ACPA identified $52.3 \%$ of patients with RF negativity as seropositive and patients with ACPA positivity had earlier presentation of disease. 


\section{LIMITATIONS}

Our study is not powered to assess the specificity of ACPA for the diagnosis of RA. Also, the long term disease outcomes and severity in relation to ACPA cannot be assessed with our study. Use of 2010 ACR/EULAR criteria might have included some undifferentiated arthritis cases as RA and thus the higher proportion of seronegative cases.

\section{REFERENCES}

1. Eggeland T, Munthe E. The role of the laboratory in rheumatology, rheumatoid factors. Rheum Dis Clin. 1983;9:135-60

2. Arnett FC, et al. The American Rheumatism Association 1987 revised criteria for the classification of rheumatoid arthritis. Arthritis Rheum. 1988; 31:315-24.

3. Nishimura K, Sugiyama D, Kogata Y, et al. Meta-analysis: diagnostic accuracy of anti-cyclic citrullinated peptide antibody and rheumatoid factor for rheumatoid arthritis. Ann Intern Med 2007; 1467-97.

4. Bizzaro N, Mazzanti G, Tonutti E, Villalta D, Tozzoli R. Diagnostic accuracy of the anti-citrulline antibody assay for rheumatoid arthritis. ClinChem 2001;47:1089-93.

5. Whiting PF, Smidt $N$, Sterne JA, et al. Systematic review: accuracy of anti-citrullinated Peptide antibodies for diagnosing rheumatoid arthritis. Ann Intern Med 2010, 152:456.

6. Avouac J, Gossec L, Dougados M. Diagnostic and predictive value of anti-cyclic citrullinated protein antibodies in rheumatoid arthritis: a systematic literature review. Ann Rheum Dis 2006; 65:845.

7. Kvien TK, Uhlig T, Ødegård S, Heiberg MS. Epidemiological aspects of rheumatoid arthritis: the sex ratio. Ann N Y Acad Sci. 2006; 1069:212-22.
8. $S$ Laivoranta-Nymana, $R$ Luukkainenb, $M$ Hakalac, $P$ Hannonend, T Möttönena, U Yli-Kerttula. Differences between female and male patients with familial rheumatoid arthritis. Ann Rheum Dis 2001;60:413-15.

9. Jawaheer D, Lum RF, Gregersen PK, Criswell LA. Influence of male sex on disease phenotype in familial rheumatoid arthritis. Arthritis Rheum 2006;54:3087-94.

10. 2010 Rheumatoid arthritis classification criteria: an American College of Rheumatology/European League Against Rheumatism collaborative initiative. Ann Rheum Dis 2010;69:1580-88.

11. Alessandri $C$, Priori $R$, Modesti M, Mancini $R$, Valesini $G$. The role of anti-cyclic cytrullinate antibodies testing in rheumatoid arthritis. Clin Rev Allergy Immunol 2008;34:45-9.

12. Toes RE, van der Woude D. ACPA (anti-citrullinated protein antibodies) and rheumatoid arthritis. ActaReumatol Port. 2011;36:205-7.

13. Kuru O, Bilgici A, Birinci A, Ulusoy H, Durupinar B. Prognostic value of anti-cyclic citrullinated peptide antibodies and rheumatoid factor in patients with rheumatoid arthritis. BratislLekListy. 2009;110:650-4.

14. Rezuş E, Grigoriu A, Rezuş C. Aggressive nature of rheumatic arthritis with citrullinated cyclic peptide antibodies. Rev Med ChirSoc Med Nat Iasi. 2009;113:73-8.

15. Predeteanu D, Varzaru L, Balanescu A, Bojinca V, Opris D, Vlad V. Anti-cyclic citrullinated peptide antibodies--activity markers in rheumatoid arthritis. JMed Life. 2009;2:36-41.

16. Lee DM, Schur PH. Clinical utility of the anti-CCP assay in patients with rheumatic diseases. Ann Rheum Dis 2003;62:870-74.

17. Vallbracht I, et al. Diagnostic and clinical value of anti-cyclic citrullinated peptide antibodies compared with rheumatoid factor isotypes in rheumatoid arthritis. Ann Rheum Dis 2004; 63:1079-84 